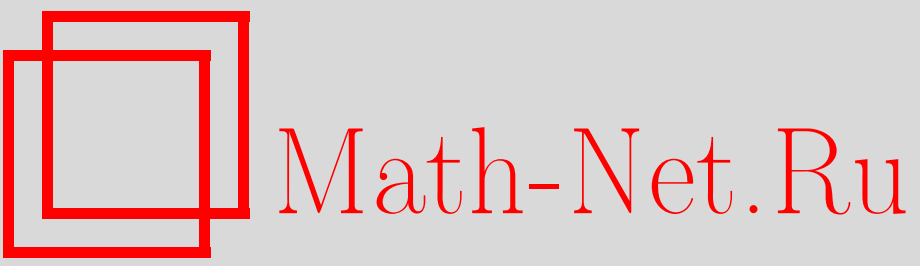

И. А. Круглов, Сходимость матриц переходных вероятностей некоторых цепей Маркова на конечной абелевой группе к равномерной матрице, Матем. вопр. криптогр., 2017, том 8, выпуск 1, 31-50

DOI: https://doi.org/10.4213/mvk213

Использование Общероссийского математического портала Math-Net.Ru подразумевает, что вы прочитали и согласны с пользовательским соглашением

http: //www . mathnet.ru/rus/agreement

Параметры загрузки:

IP : 35.173 .219 .149

26 апреля 2023 г., 13:29:12 
МАТЕМАТИЧЕСКИЕ ВОПРОСЫ КРИПТОГРАФИИ

2017 T. 8 № 1 C. 31-50

УДК 519.217.2

\title{
Сходимость матриц переходных вероятностей некоторых цепей Маркова на конечной абелевой группе к равномерной матрице
}

\author{
И. А. Круглов \\ Академия криптографии Российской Федерацฺи, Москва
}

Получено 20.IV.2015

\begin{abstract}
Аннотация. Изучается класс конечных однородных цепей Маркова, связанных со схемой авторегрессии на конечных абелевых группах. В терминах параметров схемы авторегрессии найдены условия полной регулярности и точные формулы для среднеквадратического уклонения матриц переходных вероятностей за $N$ шагов от предельной равномерной матрицы.
\end{abstract}

Ключевые слова: цепи Маркова, схема авторегрессии на конечных группах, среднеквадратическое уклонение от равномерной матрицы подстановки

Convergence of transition matrices of some Markov chains on finite Abelian group to the uniform matrix

\section{A. Kruglov}

Academy of Cryptography of the Russian Federation, Moscow

\begin{abstract}
A class of finite homogeneous Markov chains connected with the autoregression scheme on finite Abelian groups is studied. In terms of the autoregression scheme parameters some conditions of complete regularity are given and exact formulas for the mean square deviation of $N$-step transition matrices from the limiting uniform matrix are derived.
\end{abstract}

Keywords: Markov chains, autoregression scheme on finite groups, mean square deviation from the uniform matrix 


\section{Постановка задачи и формулировки результатов}

В работе исследуются конечные простые однородные цепи Маркова, определяемые следующим образом. Пусть $(G,+)$ - произвольная конечная абелева группа порядка $n \geqslant 3$ с нейтральным элементом $\theta, \alpha$ - произвольный фиксированный автоморфизм группы $G$. Образ подмножества $M \subset G$ при действии $\alpha$ будем обозначать через $M^{\alpha}$.

Рассмотрим циклическую подгруппу $\mathfrak{A}=\langle\alpha\rangle$ группы автоморфизмов группы $G$, порожденную элементом $\alpha$. Предположим, что задана последовательность $\left\{\left(\xi_{N}, \zeta_{N}\right)\right\}_{N \geqslant 1}$ независимых случайных элементов со значениями в декартовом произведении $G \times \mathfrak{A}$, члены которой распределены так же, как некоторый случайный элемент $(\xi, \zeta)$. Отметим, что случайные элементы $\xi$ и $\zeta$ могут быть зависимыми.

Определим по индукции последовательность случайных элементов со значениями в группе $G$ :

$$
\mu^{(0)}=\theta, \quad \mu^{(N)}=\left(\mu^{(N-1)}\right)^{\zeta_{N}}+\xi_{N}, \quad N \geqslant 1 .
$$

Последовательность $\left\{\mu_{N}\right\}_{N \geqslant 0}$ образует конечную простую однородную цепь Маркова с множеством состояний $G$.

Обозначим через $P=\left[p\left(\sigma, \sigma^{\prime}\right)\right]_{\sigma, \sigma^{\prime} \in G}$ матрицу переходных вероятностей цепи Маркова (1); матрица $P$ - дважды стохастическая.

Пусть $P^{N}=\left[p^{(N)}\left(\sigma, \sigma^{\prime}\right)\right]_{\sigma, \sigma^{\prime} \in G}$ есть матрица переходных вероятностей цепи Маркова (1) за $N \geqslant 1$ шагов.

Настоящая работа посвящена определению условий сходимости матриц $P^{N}$ к равномерной матрице размеров $n \times n$ :

$$
\lim _{N \rightarrow \infty} P^{N}=\left[\frac{1}{n}\right]_{n \times n},
$$

а также выводу точных формул и оценок для величин

$$
\delta_{N}^{2}=\sum_{\sigma, \sigma^{\prime} \in G}\left(p^{(N)}\left(\sigma, \sigma^{\prime}\right)-\frac{1}{n}\right)^{2}, \quad N \geqslant 1,
$$

- квадратов среднеквадратических уклонений матриц $P^{N}$ от равномерной матрицы.

В зарубежной литературе главным образом рассматривается случай группы $G$, совпадающей с аддитивной группой конечномерного векторного пространства над конечным простым полем. При этом решается задача оценки скорости сходимости распределений последовательности (1) к равномерному распределению на группе $G$ в различных частных случаях задания распределения случайного элемента $(\xi, \zeta)$. 
Бо́льшая часть работ посвящена случаю вырожденного распределения случайного элемента $\zeta$, т. е. случаю $\mathbf{P}\{\zeta=\alpha\}=1$ (см. $[6,9,10,12,13])$. В $[7,8,11]$ рассмотрен ряд случаев, в которых распределение случайного элемента $\zeta$ невырожденно и случайные элементы $\xi, \zeta$ независимы.

Оценка скорости сходимости в одном частном случае, когда случайные элементы $\xi, \zeta$ зависимы, установлена в [5]. Отметим, что условие невырожденности распределения $\zeta$ заметно усложняет исследование даже в части определения условий для выполнения равенства (2) (см. [3]).

Рассмотрим носитель $\Omega(\zeta)=\left\{\alpha_{1}, \alpha_{2}, \ldots, \alpha_{s}\right\}=\{\varphi \in \mathfrak{A} \mid \mathbf{P}\{\zeta=\varphi\}>0\}$, $s \geqslant 1$, распределения случайного элемента $\zeta$. Без ограничения общности всюду в работе предполагается, что множество $\Omega(\zeta)$ является системой порождающих элементов группы $\mathfrak{A}$.

Соотношение (2) выполняется тогда и только тогда, когда последовательность распределений случайных элементов (1) сходится при $N \rightarrow \infty$ к равномерному распределению на группе $G$. Общие условия для выполнения равенства (2) получены в [3, 4], см. также [2]. Наиболее сложным моментом в их использовании является поиск системы определяющих соотношений группы $\mathfrak{A}$ относительно порождающих элементов $\Omega(\zeta)$. В рассматриваемом случае циклической группы $\mathfrak{A}=\langle\alpha\rangle$ данная задача решается относительно просто, однако получаемые в итоге необходимые и достаточные условия для выполнения (2) остаются довольно громоздкими. Ниже будут рассмотрены некоторые частные случаи, в которых эти условия существенно упрощаются, а также возникает возможность получить для величин (3) точные формулы и верхние оценки в виде неравенств, выраженные через те или иные характеристики распределения случайного элемента $(\xi, \zeta)$.

В частности, имеет место следующее довольно общее утверждение.

Для любого элемента $\sigma \in G$ обозначим через $-\sigma$ обратный элемент к $\sigma$ в группе $G$ и положим $\tau-\sigma=\tau+(-\sigma), \quad \sigma, \tau \in G$.

Предложение 1. Предположим, что существует такой элемент $\sigma_{0} \in G$, что

$$
\mathbf{P}\left\{\xi=\sigma_{0}, \zeta=\alpha_{j}\right\}>0, \quad j=1,2, \ldots, s .
$$

Равенство (2) имеет место, если и только если не существует собственной подгруппы $L$ группы $G$, для которой выполнены соотношения

$$
\Omega(\xi) \subset \sigma_{0}+L, \quad(L)^{\alpha}=L, \quad \sigma_{0}^{\alpha_{j}}-\sigma_{0}^{\alpha_{1}} \in L, \quad j=1,2, \ldots, s .
$$

Отметим, что условие (4) выполнено, если случайные элементы $\xi$ и $\zeta$ независимы. 
Перейдем к некоторым случаям более конкретного задания $G$ и $\alpha$. Сначала будем предполагать, что конечная абелева группа $G$ не является элементарной абелевой 2 -группой, автоморфизм $\alpha$ определяется равенством

$$
\alpha(\sigma)=-\sigma, \quad \sigma \in G,
$$

и $s=2$. В этом случае $\mathfrak{A}=\Omega(\zeta)=\{\varepsilon, \alpha\}$, где $\varepsilon$-единичный автоморфизм, и можно считать, что $\alpha_{1}=\alpha, \alpha_{2}=\varepsilon, 0<\mathbf{P}\{\zeta=\alpha\}<1$.

Рассмотрим подмножества $\Omega^{(i)}=\left\{\sigma \in G \mid \mathbf{P}\left\{\xi=\sigma, \zeta=\alpha_{i}\right\}>0\right\}, i=1,2$, группы $G$, зафиксируем произвольные элементы $\sigma^{(i)} \in \Omega^{(i)}, i=1,2$, и определим подмножество

$$
M=\left(-\sigma^{(1)}+\Omega^{(1)}\right) \bigcup\left(-\sigma^{(2)}+\Omega^{(2)}\right) \bigcup\left\{2 \cdot \sigma^{(2)}\right\}
$$

элементов группы $G$. Здесь и далее $(N+1) \cdot \sigma=N \cdot \sigma+\sigma, N \geqslant 1, \sigma \in G$.

Предложение 2. Если группа $G$ не является элементарной абелевой 2-группой, автоморфизм а определяется равенством (5), то соотночение (2) выполняется тогда и только тогда, когда имеет место ровно одно из двух следующих условий:

(а) множество $M$ является системой порождающих элементов групnbl $G$,

(b) порядок группь $G$ - четное число, множество $M$ порождает подгруппу $H$ индекса 2 в группе $G$, причем $\sigma^{(1)}-\sigma^{(2)} \in G \backslash H$.

Для каждого из условий (a) и (b) в формулировке предложения 2 можно привести пример последовательности (1), для которой это условие выполнено.

Приведем точные формулы для величин $\delta_{N}^{2}$ в терминах преобразования Фурье распределения случайного элемента $\xi$ и связанных с ним условных распределений на группе $G$.

Обозначим через $(\widehat{G}, \cdot)$ группу неприводимых комплексных характеров группы $G$ с групповой операцией умножения функций, заданных на $G$. Пусть $\chi^{(0)}-$ единичный характер, $\chi^{(0)}(\sigma)=1$ при любом $\sigma \in G$. Для любого характера $\chi \in \widehat{G}$ функция $\bar{\chi}(\sigma)=\overline{\chi(\sigma)}, \sigma \in G$, также является характером. Группа $\widehat{\mathrm{G}}$ содержит в себе подгруппу $\Psi=\{\chi \in \widehat{\mathrm{G}} \mid \chi=\bar{\chi}\}$, состоящую из всех ее вещественных характеров. Число элементов этой подгруппы равно $|\Psi|=2^{k}$, где $k=0$, если $n$ нечетно, и $k$ - число слагаемых в разложении силовской 2 -подгруппы группы $G$ во внутреннюю прямую сумму примарных циклических 2-подгрупп, если $n$ четно. Пусть $\Psi=\left\{\psi_{0}, \ldots, \psi_{2^{k}-1}\right\}$, $\psi_{0}=\chi^{(0)}$. 
Так как $G$ не является элементарной абелевой 2 -группой, то $\widehat{\mathbf{G}} \backslash \Psi \neq \varnothing$, т. е. $n>2^{k}$ и $t=\left(n-2^{k}\right) / 2$ - натуральное число. Для любого неприводимого характера $\chi \in \widehat{\mathbf{G}} \backslash \Psi$ имеем $\bar{\chi} \in \widehat{\mathbf{G}} \backslash \Psi$ и $\bar{\chi} \neq \chi$. Следовательно, множество $\widehat{\mathbf{G}} \backslash \Psi$ разбивается на пары различных сопряженных характеров. Зафиксируем произвольное подмножество $\left\{\chi_{1}, \ldots, \chi_{t}\right\} \subset \widehat{\mathbf{G}} \backslash \Psi$ попарно различных характеров, для которого $\chi_{j} \neq \overline{\chi_{i}}$ при любых $1 \leqslant i<j \leqslant t$. Имеет место равенство $\widehat{\mathbf{G}}=\left\{\psi_{0}, \ldots, \psi_{2^{k}-1}, \chi_{1}, \ldots, \chi_{t}, \bar{\chi}_{1}, \ldots, \bar{\chi}_{t}\right\}$.

Рассмотрим преобразование Фурье распределения случайного элемента $\xi$ :

$$
\widehat{p}_{\xi}(\chi)=\sum_{\sigma \in G} \mathbf{P}\{\xi=\sigma\} \cdot \chi(\sigma), \quad \chi \in \widehat{\mathbf{G}},
$$

а также преобразования Фурье его условных распределений:

$$
\widehat{p}_{\xi}^{(\gamma)}(\chi)=\sum_{\sigma \in G} \mathbf{P}\left\{\xi=\sigma \mid \zeta=\alpha_{\gamma}\right) \cdot \chi(\sigma), \quad \chi \in \widehat{\mathbf{G}}, \quad \gamma=1,2 .
$$

Зафиксируем $j \in\{1, \ldots, t\}$. Пусть

$$
\mathbf{P}\{\zeta=\varepsilon\} \cdot \widehat{p}_{\xi}^{(2)}\left(\chi_{j}\right)=\omega_{j}=a_{j}+\mathbf{i} \cdot b_{j}, \quad \mathbf{P}\{\zeta=\alpha\} \cdot \widehat{p}_{\xi}^{(1)}\left(\chi_{j}\right)=\beta_{j},
$$

$\omega_{j}, \beta_{j}$ - комплексные и $a_{j}, b_{j}$ - вещественные числа, $\mathbf{i}$ - мнимая комплексная единица. Рассмотрим вещественное число $d_{j}=\left|\beta_{j}\right|^{2}-b_{j}^{2}$ и положим

$$
\lambda_{j k}=\left\{\begin{array}{lll}
a_{j}-(-1)^{k} \sqrt{d_{j}} & \text { при } & d_{j} \geqslant 0, \\
a_{j}-(-1)^{k} \mathbf{i} \cdot \sqrt{-d_{j}} & \text { при } \quad d_{j}<0,
\end{array} \quad k=1,2 .\right.
$$

Здесь $\sqrt{d}$ - арифметический квадратный корень из вещественного неотрицательного числа $d$.

Теорема 1. Для любого $N \geqslant 1$ справедлива формула

$$
\delta_{N}^{2}=\sum_{\nu=1}^{2^{k}-1}\left|\widehat{p}_{\xi}\left(\psi_{\nu}\right)\right|^{2 N}+\sum_{j=1}^{t}\left[\lambda_{j 1}^{2 N}+\lambda_{j 2}^{2 N}+\left(2 b_{j} \cdot \sum_{\nu=0}^{N-1} \lambda_{j 1}^{\nu} \cdot \lambda_{j 2}^{N-1-\nu}\right)^{2}\right]
$$

В правой части (8) в случае $k=0$ первая сумма считается равной нулю.

Введем обозначения

$$
\begin{aligned}
\varrho_{1} & = \begin{cases}\max \left\{\left|\widehat{p}_{\xi}\left(\psi_{\nu}\right)\right| \mid \nu=1, \ldots, 2^{k}-1\right\}, & k \geqslant 1, \\
0, & k=0,\end{cases} \\
\varrho_{2} & =\max \left\{\left|\lambda_{j 1}\right|,\left|\lambda_{j 2}\right| \mid j=1, \ldots, t\right\} .
\end{aligned}
$$


Так как $\left|b_{j}\right| \leqslant 1$ для любого $j=1, \ldots, t$, то из равенства (8) вытекает следующая оценка.

Следствие 1. Для любого $N \geqslant 1$ выполнено неравенство

$$
\delta_{N}^{2} \leqslant\left(2^{k}-1\right) \cdot \varrho_{1}^{2 N}+\left(n-2^{k}\right)\left(\varrho_{2}^{2}+2 N^{2}\right) \cdot \varrho_{2}^{2 N-2} .
$$

Можно показать, что при выполнении условий предложения 2 имеют место неравенства $0 \leqslant \varrho_{1}, \varrho_{2}<1$, т. е. выражение в правой части (9) стремится к нулю при $N \rightarrow \infty$.

Перейдем к рассмотрению следующего частного случая исходной постановки задачи: будем предполагать, что для некоторого простого числа $p \geqslant 2$ группа $G$ является элементарной абелевой $p$-группой.

Тогда $n=p^{m}, m \geqslant 1$, и можно считать, что $G=V_{m}(p)$ - аддитивная группа арифметического векторного пространства размерности $m$ над полем вычетов $F_{p}=\{0,1, \ldots, p-1\}$ по модулю $p$.

В данном случае автоморфизм $\alpha$ является линейным преобразованием векторного пространства $V_{m}(p)$.

Обозначим через $f_{\alpha}(\lambda)$ характеристический многочлен преобразования $\alpha$. Ввиду конечности поля $F_{p}$ свойство сепарабельности многочлена $f_{\alpha}(\lambda)$ равносильно отсутствию кратных множителей в его разложении на неприводимые над $F_{p}$ многочлены.

Введем обозначения, необходимые для формулировки условий выполнения соотношения (2). Для любого $i=1, \ldots, s$ рассмотрим множество векторов

$$
\Omega^{(i)}=\left\{\sigma \in G \mid \mathbf{P}\left\{\xi=\sigma, \zeta=\alpha_{i}\right\}>0\right\},
$$

зафиксируем произвольные элементы $\sigma_{i} \in \Omega^{(i)}, i=1, \ldots, s$, и определим множество $\Delta$ векторов из пространства $V_{m}(p)$ :

$$
\Delta=\left\{\sigma_{i}-\sigma_{i}^{\alpha_{l}}-\sigma_{l}+\sigma_{l}^{\alpha_{i}} \mid i, l=1, \ldots, s\right\} \bigcup \bigcup_{i=1}^{s}\left(\Omega^{(i)}-\sigma_{i}\right) .
$$

Занумеруем элементы данного множества: $\Delta=\left\{v_{1}, \ldots, v_{z}\right\}, z=|\Delta| \geqslant 1$. Для каждого $i=1, \ldots, z$ рассмотрим подпространство $W_{i}$ пространства $V_{m}(p)$, порожденное системой векторов $v_{i}, \ldots, v_{i}^{\alpha^{m-1}}\left(W_{i}\right.$ - циклическое подпространство вектора $v_{i}$ относительно линейного преобразования $\alpha$ ). Рассмотрим сумму подпространств

$$
W=W_{1}+\cdots+W_{z} .
$$

Заметим, что так как $W^{\alpha}=W$, то определено линейное преобразование $\bar{\alpha}$ фактор-пространства $V_{m}(p) / W:(W+v)^{\bar{\alpha}}=W+v^{\alpha}, W+v \in V_{m}(p) / W$. 
Предложение 3. Пусть группа $G$ является элементарной абелевой р-группой, многочлен $f_{\alpha}(\lambda)$ сепарабелен. Тогда для выполнения соотношения (2) необходимо и достаточно, чтобы имело место одно из следующих двух условий:

(a) $W=V_{m}(p)$,

(b) $m>1$, размерность $\operatorname{dim} V_{m}(p) / W$ фактор-пространства равна 1 , $\bar{\alpha}$ - тождественное преобразование пространства $V_{m}(p) / W, s>1$, существуют $i, j \in\{1,2, \ldots, s\}$, для которых $\sigma_{i}-\sigma_{j} \in V_{m}(p) \backslash W$.

Для каждого из условий (a), (b) предложения 3 можно привести пример последовательности (1), для которой это условие выполнено.

Следствие 2. Пусть группа $G$ является элементарной абелевой р-группой, многочлен $f_{\alpha}(\lambda)$ сепарабелен, $f_{\alpha}(1) \neq 0$. Тогда для выполнения соотномения (2) необходимо и достаточно, чтобы $W=V_{m}(p)$.

Следствие 3. Пусть группа $G$ является элементарной абелевой р-группой и многочлен $f_{\alpha}(\lambda)$ неприводим. Тогда для выполнения соотношения (2) необходимо и достаточно, чтобы множество $\Delta$ содержало хотя бы один ненулевой вектор.

Проводя оценку величин (3), будем дополнительно предполагать, что характеристический многочлен линейного преобразования $\alpha$ является неприводимым примитивным многочленом над полем $F_{p}$. В этом случае $|\mathfrak{A}|=p^{m}-1$.

Введем обозначение $p_{g, i}=\mathbf{P}\left\{\xi=g, \zeta=\alpha_{i}\right\}, g \in V_{m}(p), i=1, \ldots, s$, для элементов распределения $(\xi, \zeta)$ и обозначение $q_{i}=\mathbf{P}\left\{\zeta=\alpha_{i}\right\}, i=1, \ldots$ $\ldots, s$, для элементов распределения $\zeta$. Положим

$$
\rho=p^{m} \cdot \sum_{g \in V_{m}(p)} \sum_{i=1}^{s} p_{g, i}^{2}-\sum_{i=1}^{s} q_{i}^{2} ;
$$

можно показать, что $\rho \geqslant 0$.

Теорема 2. Пусть $G$ - элементарная абелева р-группа и характеристический многочлен линейного преобразования $\alpha$ - неприводимый примитивный многочлен над полем $F_{p}$.

Тогда имеют место неравенства

$$
\delta_{N} \leqslant \rho^{N}, \quad N \geqslant 1 .
$$




\section{Доказательства результатов}

Начнем с условий выполнения соотношения (2) (предложения 1, 2, 3).

Так как группа $\mathfrak{A}$ коммутативна, то предложение 1 является частным случаем следствия 3 из [4].

В условиях предложения 2 имеем следующую систему определяющих соотношений группы $\mathfrak{A}$ относительно порождающих элементов $\Omega(\zeta)=\left\{\alpha_{1}, \alpha_{2}\right\}$ :

$$
\alpha_{1}^{2}=\alpha_{2}=\varepsilon
$$

Используя это обстоятельство, можно получить утверждение предложения 2 непосредственным применением теоремы 1 из [3].

Доказательство предложения 3. Воспользуемся рассуждениями, использованными в $[2,3]$. В соответствии с ними, исходя из носителя распределения $\Omega(\xi, \zeta)$, можно определить подгруппы $H$ и $G_{1}$ группы $G$, а также подгруппы $\mathfrak{N}$ и $\mathfrak{B}$ группы $\mathfrak{A}$, для которых справедливы, в частности, следующие соотношения: $H^{\alpha}=H, H \subset G_{1}, \mathfrak{B} \subset \mathfrak{N}$.

Кроме того, в [3] определяется некоторый гомоморфизм $\varphi: \mathfrak{B} \rightarrow G_{1} / H$.

В терминах этих подгрупп и гомоморфизма в следствии 1 из [3] сформулирован следующий критерий: необходимым и достаточным условием для выполнения соотношения (2) является одновременное выполнение равенства $\varphi(\mathfrak{B})=G_{1} / H$ и равенства $\left|G: G_{1}\right|=|\mathfrak{N}: \mathfrak{B}|$ для индексов подгрупп.

Порядок группы $\mathfrak{A}$ равен периоду многочлена $f_{\alpha}(\lambda)$; вследствие сепарабельности последнего этот период является делителем числа $p^{N_{0}}-1$ для некоторого натурального $N_{0}$. Поэтому $|\mathfrak{A}|$ не делится на $p$.

Следовательно, и числа $|\mathfrak{N}: \mathfrak{B}|,|\varphi(\mathfrak{B})|$ не делятся на $p$.

Однако числа $\left|G: G_{1}\right|$ и $\left|G_{1} / H\right|$ есть степени числа $p$. Таким образом, в нашем случае необходимым и достаточным условием для выполнения (2) является равенство $H=G$. Отсюда следует, что для доказательства предложения достаточно обосновать, что равенство

$$
H=V_{m}(p)
$$

выполняется тогда и только тогда, когда выполнено одно из условий (a), (b) формулировки предложения.

Докажем сначала ряд фактов, верных независимо от выполнения этих условий. Согласно доказательству теоремы из $§ 4$ в [3] подгруппа $H$ обладает следующими свойствами. 
Рассмотрим произвольные $N \geqslant 1$ и $\varphi \in \Omega\left(\zeta_{1} \cdot \ldots \cdot \zeta_{N}\right)$, обозначим через $\Omega_{\varphi}\left(\mu^{(N)}\right)$ носитель условного распределения случайного элемента $\mu^{(N)}$ при условии $\zeta_{1} \cdot \ldots \cdot \zeta_{N}=\varphi$. Для любого элемента $\tau \in \Omega_{\varphi}\left(\mu^{(N)}\right)$ справедливо включение

$$
\Omega_{\varphi}\left(\mu^{(N)}\right) \subset \tau+H
$$

и существует такое $N^{\prime} \geqslant 1$, что при любом $N \geqslant N^{\prime}$ в соотношении (12) выполняется равенство. Далее, рассмотрим значения $N \geqslant 1$, удовлетворяющие следующему условию: $\varepsilon \in \Omega\left(\zeta_{1} \cdot \ldots \cdot \zeta_{N}\right)$. Известно (см., например, [1]), что эти значения $N$ образуют некоторую арифметическую прогрессию с разностью $d \geqslant 1$, первый член которой делится на $d$. При этом $d-$ делитель периода преобразования $\alpha$, следовательно, $d$ делит $p^{N_{0}}-1$. Существует такой вектор $x \in V_{m}(p)$, что

$$
\Omega_{\varepsilon}\left(\mu^{(N d)}\right)=N \cdot x+H, \quad N \geqslant N^{\prime} .
$$

Докажем соотношение

$$
W \subset H .
$$

Для любого $i \in\{1, \ldots, s\}$ из соотношения (12) при $N=1, \varphi=\alpha_{i}$ следует включение $\Omega^{(i)} \subset \sigma_{i}+H$. Для любых $i, l \in\{1, \ldots, s\}$ из (12) при $N=2$, $\varphi=\alpha_{i} \cdot \alpha_{l}=\alpha_{l} \cdot \alpha_{i}$ вытекает равенство

$$
\sigma_{i}+\sigma_{l}^{\alpha_{i}}+H=\sigma_{l}+\sigma_{i}^{\alpha_{l}}+H .
$$

Следовательно, $\Delta \subset H$.

Так как поле $F_{p}$ - простое, то подгруппа $H$ является подпространством пространства $V_{m}(p)$, инвариантным относительно действия $\alpha$.

Из включения $\Delta \subset H$ следует, что $W \subset H$.

Используя (13), покажем, что для любого $\tau \in H$ имеет место равенство

$$
W+\tau^{\alpha}=W+\tau .
$$

Рассмотрим произвольный элемент $\tau \in H$. Возьмем в (13) значение $N=N^{\prime} p$ и положим $K=N^{\prime} p d$. Получим равенство

$$
\Omega_{\varepsilon}\left(\mu^{(K)}\right)=H .
$$

В этом случае существует реализация $\left(\sigma_{i_{1}}, \alpha_{i_{1}}\right), \ldots,\left(\sigma_{i_{K}}, \alpha_{i_{K}}\right)$ случайной последовательности $\left\{\left(\xi_{l}, \zeta_{l}\right)\right\}_{l=1}^{K}$, для которой $\alpha_{i_{1}} \cdot \ldots \cdot \alpha_{i_{K}}=\varepsilon$, и соответствующее этой реализации значение случайного элемента $\mu^{(K)}$ равно $\tau$, т.е.

$$
\tau=\sigma_{i_{K}}+\sigma_{i_{K-1}}^{\alpha_{i_{K}}}+\sigma_{i_{K-2}}^{\alpha_{i_{K-1}} \cdot \alpha_{i_{K}}}+\ldots+\sigma_{i_{2}}^{\alpha_{i_{3}} \cdot \ldots \cdot \alpha_{i_{K}}}+\sigma_{i_{1}}^{\alpha_{i_{2}} \cdot \ldots \cdot \alpha_{i_{K}}}
$$


Используя коммутативность группы $V_{m}(p)$, соотношения

$$
\begin{aligned}
W^{\alpha} & =W \\
W+\sigma_{i}-\sigma_{i}^{\alpha_{l}} & =W+\sigma_{l}-\sigma_{l}^{\alpha_{i}}, \\
\Omega^{(i)} & \subset \sigma_{i}+W, \quad i, l=1, \ldots, s,
\end{aligned}
$$

а также свойство перестановочности преобразований $\alpha_{1}, \alpha_{2}, \ldots, \alpha_{s}$, получим: для любого $l=1, \ldots, s$

$$
\begin{aligned}
W+\tau-\tau^{\alpha_{l}}= & W+\left(\sigma_{i_{K}}-\sigma_{i_{K}}^{\alpha_{l}}\right)+ \\
& +\left(\sigma_{i_{K-1}}-\sigma_{i_{K-1}}^{\alpha_{l}}\right)^{\alpha_{i_{K}}}+\left(\sigma_{i_{K-2}}-\sigma_{i_{K}-2}^{\alpha_{l}}\right)^{\alpha_{i_{K}-1} \cdot \alpha_{i_{K}}}+\cdots \\
& \cdots+\left(\sigma_{i_{2}}-\sigma_{i_{2}}^{\alpha_{l}}\right)^{\alpha_{i_{3}} \cdots \cdot \alpha_{i_{K}}}+\left(\sigma_{i_{1}}-\sigma_{i_{1}}^{\alpha_{l}}\right)^{\alpha_{i_{2}} \cdot \ldots \cdot \alpha_{i_{K}}}= \\
= & W+\left(\sigma_{l}-\sigma_{l}^{\alpha_{i_{K}}}\right)+\left(\sigma_{l}-\sigma_{l}^{\alpha_{i_{K}-1}}\right)^{\alpha_{i_{K}}}+ \\
& +\left(\sigma_{l}-\sigma_{l}^{\alpha_{i_{K}-2}}\right)^{\alpha_{i_{K-1}} \cdot \alpha_{i_{K}}}+\cdots \\
& \cdots+\left(\sigma_{l}-\sigma_{l}^{\alpha_{i_{2}}}\right)^{\alpha_{i_{3}} \cdot \ldots \cdot \alpha_{i_{K}}}+\left(\sigma_{l}-\sigma_{l}^{\alpha_{i_{1}}}\right)^{\alpha_{i_{2}} \cdot \ldots \cdot \alpha_{i_{K}}}= \\
= & W+\sigma_{l}-\sigma_{l}^{\varepsilon}=W \\
(W+\tau)^{\alpha_{l}}= & W+\tau, \quad l=1, \ldots, s .
\end{aligned}
$$

Так как $\alpha_{1}, \alpha_{2}, \ldots, \alpha_{s}-$ множество порождающих элементов группы $\mathfrak{A}=\langle\alpha\rangle$, то отсюда следует (15).

Перейдем к доказательству эквивалентности условия (2) одному из условий (a), (b) формулировки предложения 3.

Рассмотрим случай, когда $m=1$, т.е. $V_{m}(p)=F_{p}$. Если $W=F_{p}$, то из (14) следует (11). Пусть верно (11), но $W \neq F_{p}$. В этом случае $W=\{\theta\}$, $\Delta=\{\theta\}$, и из (15) следует равенство $\alpha=\varepsilon$. Но тогда

$$
s=1, \quad \Delta=\Omega(\xi) \backslash \sigma_{1}, \quad \Omega(\xi)=\left\{\sigma_{1}\right\}
$$

и

$$
\Omega\left(\mu^{(N)}\right)=\left\{N \cdot \sigma_{1}\right\} \quad \text { при любом } \quad N \geqslant 1 .
$$

Учитывая соотношение (13), получаем противоречие с предположением (11). Итак, в случае $m=1$ выполнение условия (2) эквивалентно условию (а).

Рассмотрим случай, когда $f_{\alpha}(1) \neq 0$, значение $m \geqslant 1$ произвольное. Покажем, что имеет место равенство $H=W$. 
Если $W=F_{p}$, то это равенство следует из (14). Пусть $W \neq F_{p}$, тогда фактор-пространство $V_{m}(p) / W$ - ненулевое. Характеристический многочлен $f_{\bar{\alpha}}(\lambda)$ линейного преобразования $\bar{\alpha}$ является делителем многочлена $f_{\alpha}(\lambda)$, значит, $f_{\bar{\alpha}}(1) \neq 0$. Поэтому равенство (15) может выполняться лишь в случае, когда $\tau \in W$.

Ввиду произвольности выбора элемента $\tau \in H$ получаем включение $H \subset W$, т. е. $H=W$. С учетом сказанного выше в случае $f_{\alpha}(1) \neq 0$ выполнение условия (2) эквивалентно равенству $W=V_{m}(p)$, т. е. вновь эквивалентно условию (а).

Рассмотрим случай, когда $m>1, f_{\alpha}(1)=0$. Если при этом $W=V_{m}(p)$, то и равенство (2), и условие (а) выполняются. Предположим дополнительно, что $W \neq V_{m}(p)$. Покажем, что тогда выполнение условия (2) эквивалентно условию (b).

Если имеет место равенство (2), то верно (11), и из соотношения (15) следует, что $\bar{\alpha}=\bar{\varepsilon}$ есть тождественное преобразование пространства $V_{m}(p) / W$. В этом случае

$$
f_{\bar{\alpha}}(\lambda)=(\lambda-1)^{w}, \quad \text { где } \quad w=\operatorname{dim} V_{m}(p) / W .
$$

Так как $f_{\bar{\alpha}}(\lambda)$ делит сепарабельный многочлен $f_{\alpha}(\lambda)$, то $w=1$. Предположим, что

$$
\sigma_{i}-\sigma_{j} \in W \quad \text { для любых } \quad i, j \in\{1,2, \ldots, s\} .
$$

Так как $\Omega^{(i)} \subset \sigma_{i}+W, i=1,2, \ldots, s$, то $\Omega(\xi) \subset \sigma_{1}+W$. Ввиду равенства $\bar{\alpha}=\bar{\varepsilon}$ и соотношения (1) отсюда следует включение

$$
\Omega\left(\mu^{(N)}\right) \subset N \cdot \sigma_{1}+W \quad \text { при любом } \quad N \geqslant 1 .
$$

Последнее противоречит равенствам (11), (13). Таким образом, существуют $i, j \in\{1,2, \ldots, s\}$, для которых $\sigma_{i}-\sigma_{j} \in V_{m}(p) \backslash W$, и условие (b) выполнено.

Наконец, пусть выполнено условие (b), докажем, что верно (11). Порядки линейных преобразований $\alpha_{i}, \alpha_{j}$, как и порядок $\alpha$, делят $p^{N_{0}}-1$. Следовательно, эти порядки делят число $p^{N N_{0}}-1$ при любом $N \geqslant 1$. Выберем значение $N$, для которого

$$
l=\frac{1}{d}\left(p^{N N_{0}}-1\right)>N^{\prime}
$$

2017, T. 8, № 1, C. 31-50 
C таким натуральным числом $l$ из выполнения условия (b) и соотношений (14), (13) следуют включения

$$
\begin{aligned}
\left(p^{N N_{0}}-1\right) \cdot \sigma_{i}+W & \subset \Omega_{\varepsilon}\left(\mu^{(l d)}\right)+W, \\
\left(p^{N N_{0}}-1\right) \cdot \sigma_{j}+W & \subset \Omega_{\varepsilon}\left(\mu^{(l d)}\right)+W, \\
- & \sigma_{i}+W \subset l \cdot x+H, \\
- & \sigma_{j}+W \subset l \cdot x+H .
\end{aligned}
$$

Значит, $\sigma_{i}-\sigma_{j} \in H \backslash W$. Из условия (14) и равенства $\operatorname{dim} V_{m}(p) / W=1$ следует равенство (11).

Предложение 3 доказано полностью. В ходе доказательства показано, что в случаях, когда $m=1$ или $f_{\alpha}(1) \neq 0$, выполнение условия (2) равносильно условию (а) формулировки предложения. Отсюда вытекают утверждения следствия 2 и следствия 3.

Для исследования величин (3) представим матрицу $P^{N}$ при любом $N \geqslant 1$ как матрицу переходных вероятностей, соответствующую произведению $N$ независимых случайных элементов со значениями в некоторой группе подстановок $\mathfrak{F}$ множества элементов группы $G$ (см. [2]). Группа $\mathfrak{A}$ является подгруппой симметрической группы $(\mathfrak{S}(G), \cdot)$ подстановок множества $G$. Для любого элемента $g \in G$ обозначим через $\widetilde{g} \in \mathfrak{S}(G)$ соответствующий сдвиг группы $G$ :

$$
\sigma^{\widetilde{g}}=\sigma+g, \quad \sigma \in G .
$$

Пусть $\mathfrak{G}=\{\widetilde{g} \mid g \in G\}-$ подгруппа группы $\mathfrak{S}(G)$, состоящая из всех сдвигов. Так как $\mathfrak{A} \cdot \mathfrak{G}=\mathfrak{G} \cdot \mathfrak{A}$, то $\mathfrak{F}=\mathfrak{G} \cdot \mathfrak{A}-$ подгруппа в $\mathfrak{S}(G)$. Будучи группой подстановок, группа $\mathfrak{F}$ определяет свое подстановочное представление с областью действия $G$; это представление обозначим через $q$. Представление $q$ транзитивно.

Пусть $Q$ - линейное представление группы $\mathfrak{F}$ подстановочными матрицами, соответствующее подстановочному представлению $q$. Для произвольного случайного элемента $\eta$ со значениями в группе $\mathfrak{F}$ определена матрица

$$
\Pi_{\eta}^{(q)}=\sum_{\varphi \in \mathfrak{F}} \mathbf{P}\{\eta=\varphi\} \cdot Q(\varphi)
$$

переходных вероятностей размера $n \times n$. Пусть $U_{0}, U_{1}, \ldots, U_{r}-$ некоторая фиксированная совокупность попарно неэквивалентных неприводимых унитарных комплексных матричных представлений группы $G$, являющихся неприводимыми компонентами представления $Q$, причем $U_{0}$ - единичное представление группы $G$, и для каждой неприводимой компоненты $U$ представления $Q$ существует $j \in\{0,1, \ldots, r\}$, для которого $U_{j}$ эквивалентно $U$. 
Для каждого $j \in\{0,1, \ldots, r\}$ обозначим через $k^{(j)}$ кратность вхождения представления $U_{j}$ в представление $Q$. Соотношение

$$
\Phi_{\eta}^{(q)}\left(U_{j}\right)=\sum_{\varphi \in \mathfrak{F}} \mathbf{P}\{\eta=\varphi\} \cdot Q(\varphi), \quad j=0,1, \ldots, r
$$

определяет матричную характеристическую функцию матрицы $\Pi_{\eta}^{(q)}$ (см. [2]).

Изоморфизм

$$
\pi: G \rightarrow \mathfrak{G}, \quad \pi(g)=\widetilde{g}, \quad g \in G,
$$

есть регулярное представление абелевой группы $G$. Для любого $N \geqslant 1$ рассмотрим случайные элементы $\zeta_{N}, \pi\left(\xi_{N}\right)$ со значениями в группе $\mathfrak{F}$ и положим

$$
\eta_{N}=\left(\zeta_{1} \cdot \pi\left(\xi_{1}\right)\right) \cdot \ldots \cdot\left(\zeta_{N} \cdot \pi\left(\xi_{N}\right)\right), \quad N \geqslant 1 .
$$

Случайный элемент $\eta_{N}$ является произведением $N$ независимых одинаково распределенных случайных элементов, распределение которых совпадает с распределением $\eta_{1}$. Для произвольной комплексной квадратной матрицы $C=\left[c_{i, j}\right]$ введем ее евклидову норму:

$$
\epsilon(C)=\left(\sum_{i, j}\left|c_{i, j}\right|^{2}\right)^{1 / 2}
$$

Имеет место равенство

$$
\delta_{N}^{2}=\epsilon\left(P^{N}-\left[\frac{1}{n}\right]\right)^{2} .
$$

Из результатов работы [2] вытекает следующее утверждение.

Лемма 1. Для любого $N \geqslant 1$ выполнены равенства

$$
\begin{gathered}
P^{N}=\Pi_{\eta_{N}}^{(q)}, \\
\delta_{N}^{2}=\sum_{j=1}^{r} k^{(j)} \cdot \epsilon\left(\Phi_{\eta_{1}}^{(q)}\left(U_{j}\right)^{N}\right)^{2} .
\end{gathered}
$$

Использование соотношений (18) и (19) при доказательстве теорем 1, 2 основано «свойствах минимальности» представления $Q:$ в условиях теоремы 1 степени неприводимых компонент $Q$ равны 1 или 2, в условиях теоремы 2 представление $Q$ имеет единственную неединичную неприводимую компоненту. В обоих случаях удается получить удобные для расчетов формулы для элементов матриц неприводимых компонент. 
Общая идея применения соотношений (18) и (19) при оценке скорости сходимости матриц переходных вероятностей цепей Маркова к предельной равномерной матрице принадлежит Ю. Н. Горчинскому (см. [2]). В зарубежной литературе аналогичные методы развивались в работах Р. Грэхема, П. Диакониса и других авторов (см. [6]). Идея использования указанных «свойств минимальности» представления $Q$ применялась в работах А.В. Подрябинникова, опубликованных в ведомственном издании в середине 1970-х годов.

Теоремы 1, 2 настоящей работы можно рассматривать как развитие данного направления исследований.

Доказательство теоремы 1. Для того чтобы использовать равенство (19), дадим описание представлений $U_{j}$ и чисел $k^{(j)}, j \in\{0,1, \ldots, r\}$. Исходя из изоморфизма $\pi$, мы не будем различать характеры групп $G$ и $\mathfrak{G}$, полагая $\widehat{\mathfrak{G}}=\widehat{G}$ и

$$
\chi(\widetilde{g})=\chi(g), \quad g \in G, \quad \chi \in \widehat{\mathbf{G}} .
$$

Опишем одномерные компоненты $Q$.

Для каждого $j=0, \ldots, 2^{k}-1$ определим одномерное линейное представление $U_{j}$ группы $\mathfrak{F}$, положив для любого $g \in G$

$$
U_{j}(\widetilde{g})=U_{j}(\widetilde{g} \cdot \alpha)=\psi_{j}(g) .
$$

Можно показать, что для характера $\chi_{Q}$ представления $Q$ имеет место формула

$$
\chi_{Q}\left(\widetilde{g} \cdot \alpha^{\gamma}\right)=\left\{\begin{array}{clc}
2 n, & g=\theta, & \gamma=0, \\
2^{k}, & g \in 2 \cdot G, & \gamma=1, \\
0, & g \in G \backslash \theta, & \gamma=0, \\
0, & g \in G \backslash 2 \cdot G, & \gamma=1 .
\end{array}\right.
$$

Введем скалярное произведение комплексных функций, заданных на группе $\mathfrak{F}$ :

$$
\left(\omega_{1}, \omega_{2}\right)=\frac{1}{|\mathfrak{F}|} \sum_{\varphi \in \mathfrak{F}} \omega_{1}(\varphi) \cdot \overline{\omega_{2}(\varphi)}
$$

Имеют место равенства

$$
\left(U_{j}, U_{j}\right)=\left(U_{j}, \chi_{Q}\right)=1 \text {. }
$$

Следовательно, представления $U_{0}, \ldots, U_{2^{k}-1}$ являются попарно неэквивалентными неприводимыми одномерными компонентами представления $Q$.

Опишем двумерные компоненты $Q$. 
Для любого $j=1, \ldots, t$ рассмотрим двумерное представление $W_{j}$, которое является представлением группы $\mathfrak{F}$, индуцированным представлением $\chi_{j}$ ее подгруппы $\mathfrak{G}$. Для любого $\widetilde{g} \in \mathfrak{G}$ справедливы равенства

$$
W_{j}(\widetilde{g})=\left(\begin{array}{cc}
\chi_{j}(g) & 0 \\
0 & \chi_{j}(g)
\end{array}\right), \quad W_{j}(\widetilde{g} \cdot \alpha)=\left(\begin{array}{cc}
0 & \chi_{j}(g) \\
\chi_{j}(g) & 0
\end{array}\right) .
$$

Для характера $\chi_{W_{j}}$ представления $W_{j}$ справедливо соотношение

$$
\chi_{W_{j}}\left(\widetilde{g} \cdot \alpha^{\gamma}\right)= \begin{cases}\chi_{j}(g)+\overline{\chi_{j}(g)}, & \gamma=0 \\ 0, & \gamma=1\end{cases}
$$

Имеют место равенства

$$
\left(\chi_{W_{j}}, \chi_{W_{j}}\right)=\left(\chi_{W_{j}}, \chi_{Q}\right)=1, \quad j=1, \ldots, t, \quad\left(\chi_{W_{j}}, \chi_{W_{j}}\right)=0, \quad 1 \leqslant l<j \leqslant t .
$$

Следовательно, представления $W_{j}, j=1, \ldots, t$, являются попарно неэквивалентными неприводимыми двумерными компонентами представления $Q$.

Положим

$$
r^{\prime}=2^{k}-1+t, \quad U_{2^{k}-1+j}=W_{j}, \quad j=1, \ldots, t .
$$

Неприводимые представления $U_{0}, \ldots, U_{r}$ попарно неэквивалентны и являются компонентами представления $Q$. Суммарная степень этих представлений равна степени представления $Q$. Следовательно, $r=r^{\prime}, Q$ не имеет других неприводимых компонент и кратности компонент $U_{0}, \ldots, U_{r}$ в представлении $Q$ равны

$$
k^{(j)}=1, \quad j=0,1, \ldots, r .
$$

Из соотношений (17), (20), (6) следует равенство

$$
\Phi_{\eta_{1}}^{(q)}\left(U_{j}\right)=\widehat{p}_{\xi_{1}}\left(\psi_{j}\right), \quad j=1, \ldots, 2^{k}-1, \quad N \geqslant 1,
$$

из которого вытекает следующее утверждение.

Лемма 2. Если $k \geqslant 1$, то для любых $j=1, \ldots, 2^{k}-1 u N \geqslant 1$ имеет место равенство

$$
\epsilon\left(\Phi_{\eta_{1}}^{(q)}\left(U_{j}\right)^{N}\right)^{2}=\left|\widehat{p}_{\xi_{1}}\left(\psi_{j}\right)\right|^{2 N}
$$

Для величин $\epsilon\left(\Phi_{\eta_{1}}^{(q)}\left(U_{j}\right)^{N}\right)^{2}$ при $j=2^{k}, \ldots, r, N \geqslant 1$ справедливо следующее утверждение.

Лемма 3. Для любых $j=2^{k}, \ldots, r$ u $N \geqslant 1$ имеет место равенство

$$
\epsilon\left(\Phi_{\eta_{1}}^{(q)}\left(U_{j}\right)^{N}\right)^{2}=\lambda_{j 1}^{2 N}+\lambda_{j 2}^{2 N}+\left(2 \cdot b_{j} \cdot \sum_{\nu=0}^{N-1} \lambda_{j 1}^{\nu} \cdot \lambda_{j 2}^{N-1-\nu}\right)^{2} .
$$


Доказательство леммы 3. Из соотношений (17), (21), (23), (7), (8) следует равенство

$$
\Phi_{\eta_{1}}^{(q)}\left(U_{j}\right)=\left(\begin{array}{ll}
\omega_{j} & \beta_{j} \\
\overline{\beta_{j}} & \overline{\omega_{j}}
\end{array}\right) .
$$

Можно непосредственно показать, что $\lambda_{j 1}, \lambda_{j 2}$ - собственные числа матрицы (26).

Далее при доказательстве равенства (25) с помощью жордановой формы матриц (26) можно выделить следующие случаи:

$$
\begin{aligned}
& \beta_{j}=0, \\
\beta_{j} \neq 0, & d_{j}=0, \\
\beta_{j} \neq 0, & d_{j}>0, \\
\beta_{j} \neq 0, & d_{j}<0 .
\end{aligned}
$$

Приведем доказательство для последнего случая, когда $\beta_{j} \neq 0, d_{j}<0$. Здесь $\lambda_{j 1}=a_{j}+\mathbf{i} \cdot \sqrt{-d_{j}}, \lambda_{j 2}=a_{j}-\mathbf{i} \cdot \sqrt{-d_{j}}-$ мнимые числа, $\lambda_{j 1}=\overline{\lambda_{j 2}}$. Матрица

$$
C=\left(\begin{array}{cc}
\beta_{j} & \beta_{j} \\
\mathbf{i} \cdot\left(\sqrt{-d_{j}}-b_{j}\right) & \mathbf{i} \cdot\left(-\sqrt{-d_{j}}-b_{j}\right)
\end{array}\right)
$$

обратима, ее определитель $|C|$ равен $-2 \mathbf{i} \cdot \beta_{j} \sqrt{-d_{j}}$ и

$$
C^{-1}=\frac{1}{2 \beta_{j} \sqrt{-d_{j}}}\left(\begin{array}{cc}
\sqrt{-d_{j}}+b_{j} & -\mathbf{i} \cdot \beta_{j} \\
\sqrt{-d_{j}}-b_{j} & \mathbf{i} \cdot \beta_{j}
\end{array}\right) .
$$

Имеет место равенство

$$
\Phi_{\eta_{1}}^{(q)}\left(U_{j}\right)=C \cdot\left(\begin{array}{cc}
\lambda_{j 1} & 0 \\
0 & \lambda_{j 2}
\end{array}\right) \cdot C^{-1}
$$

поэтому

$$
\Phi_{\eta_{1}}^{(q)}\left(U_{j}\right)^{N}=\frac{1}{2 \sqrt{-d_{j}}} \cdot\left(\begin{array}{ll}
f_{11} & f_{12} \\
f_{21} & f_{22}
\end{array}\right),
$$

где

$$
\begin{aligned}
& f_{11}=\lambda_{j 1}^{N} \cdot\left(\sqrt{-d_{j}}+b_{j}\right)+\lambda_{j 2}^{N} \cdot\left(\sqrt{-d_{j}}-b_{j}\right), \\
& f_{12}=\mathbf{i} \cdot \beta_{j} \cdot\left(\lambda_{j 2}^{N}-\lambda_{j 1}^{N}\right), \\
& f_{21}=\mathbf{i} \cdot \frac{\left|\beta_{j}\right|^{2}}{\beta_{j}} \cdot\left(\lambda_{j 2}^{N}-\lambda_{j 1}^{N}\right), \\
& f_{22}=\lambda_{j 1}^{N} \cdot\left(\sqrt{-d_{j}}-b_{j}\right)+\lambda_{j 2}^{N} \cdot\left(\sqrt{-d_{j}}+b_{j}\right) .
\end{aligned}
$$


Следовательно,

$$
\begin{aligned}
\epsilon\left(\Phi_{\eta_{1}}^{(q)}\left(U_{j}\right)^{N}\right)^{2}= & \frac{1}{-4 d_{j}} \cdot\left(\left|f_{11}\right|^{2}+\left|f_{12}\right|^{2}+\left|f_{21}\right|^{2}+\left|f_{22}\right|^{2}\right)= \\
= & {\left[2\left(\lambda_{j 1}^{N}\left(\sqrt{-d_{j}}+b_{j}\right)+\lambda_{j 2}^{N}\left(\sqrt{-d_{j}}-b_{j}\right)\right) \cdot\right.} \\
& \cdot\left(\lambda_{j 2}^{N}\left(\sqrt{-d_{j}}+b_{j}\right)+\lambda_{j 1}^{N}\left(\sqrt{-d_{j}}-b_{j}\right)\right)+ \\
& \left.\quad+2\left|\beta_{j}\right|^{2} \cdot\left(\lambda_{j 2}^{N}-\lambda_{j 1}^{N}\right) \cdot\left(\lambda_{j 1}^{N}-\lambda_{j 2}^{N}\right)\right] \cdot \frac{1}{-4 d_{j}}= \\
= & \frac{1}{d_{j}} \cdot\left[\left(\lambda_{j 1}^{2 N}+\lambda_{j 2}^{2 N}\right) \cdot\left|\beta_{j}\right|^{2}-2\left(\lambda_{j 1} \cdot \lambda_{j 2}\right)^{N} \cdot b_{j}^{2}\right]= \\
= & \lambda_{j 1}^{2 N}+\lambda_{j 2}^{2 N}+\left(2 b_{j} \cdot \sum_{\nu=0}^{N-1} \lambda_{j 1}^{\nu} \cdot \lambda_{j 2}^{N-1-\nu}\right)^{2} .
\end{aligned}
$$

Утверждение теоремы 1 является следствием лемм 1, 2 и 3.

Доказательство теоремы 2. По условию теоремы $\alpha$-линейное преобразование максимального периода. Так как стабилизатор $\mathfrak{A}=\langle\alpha\rangle$ точки $\theta-$ транзитивная группа на множестве $V_{p}(p) \backslash \theta$, то группа подстановок $\mathfrak{F}$ является дважды транзитивной. В этом случае $r=1$. Единственную неединичную неприводимую компоненту степени $p^{m}-1$ представления $Q$ с точностью до эквивалентности можно задать следующим образом.

Элементы $\left\{\varepsilon, \alpha, \ldots, \alpha^{p^{m}-2}\right\}$ образуют полную систему представителей смежных классов группы $\mathfrak{F}$ по подгруппе $\mathfrak{G}$. Зафиксируем произвольный неединичный характер $\psi$ группы $\mathfrak{G}$ и, используя выбранную систему представителей смежных классов, вычислим матричное представление $U$ группы $\mathfrak{F}$, индуцированное представлением $\psi$ ее подгруппы $\mathfrak{G}$. Для любых $\widetilde{g} \in \mathfrak{G}$ и $\nu=0,1, \ldots, p^{m}-2$ имеют место равенства

$$
\begin{aligned}
U\left(\widetilde{g} \cdot \alpha^{\nu}\right) & =\left[U_{l, j}\left(\widetilde{g} \cdot \alpha^{\nu}\right)\right]_{l, j=1}^{p^{m}-1}, \\
U_{l, j}\left(\widetilde{g} \cdot \alpha^{\nu}\right) & = \begin{cases}\psi\left(\alpha^{l} \cdot \widetilde{g} \cdot \alpha^{\nu-j}\right), & \alpha^{l} \cdot \widetilde{g} \cdot \alpha^{\nu-j} \in \mathfrak{G}, \\
0, & \alpha^{l} \cdot \widetilde{g} \cdot \alpha^{\nu-j} \in \mathfrak{F} \backslash \mathfrak{G},\end{cases}
\end{aligned}
$$

где $l, j=1, \ldots, p^{m}-1$.

2017, T. 8, № 1, C. $31-50$ 
Для любого $l=1, \ldots, p^{m}-1$ равенства

$$
\psi^{(l)}(g)=\psi\left(\widetilde{g^{\left(\alpha^{-l}\right)}}\right), \quad g \in G,
$$

определяют характер группы $G$, и выполняется равенство

$$
\widehat{G}=\left\{\chi^{(0)}, \psi^{(0)}, \ldots, \psi^{\left(p^{m}-2\right)}\right\} .
$$

Так как $\alpha^{l} \cdot \widetilde{g} \cdot \alpha^{\nu-j}=\widetilde{g^{\left(\alpha^{-l}\right)}} \cdot \alpha^{\nu+l-j}$, то для любых $l, j=1, \ldots, p^{m}-1$

$$
U_{l, j}\left(\widetilde{g} \cdot \alpha^{\nu}\right)= \begin{cases}\psi^{(l)}(g), & j=l+\nu\left(\bmod \left(p^{m}-1\right)\right), \\ 0, & j \neq l+\nu\left(\bmod \left(p^{m}-1\right)\right) .\end{cases}
$$

Для характеров представлений $Q$ и $U$ имеют место равенства

$$
\begin{aligned}
& \chi_{Q}\left(\widetilde{g} \cdot \alpha^{\nu}\right)=\left\{\begin{array}{rll}
p^{m}, & g=\theta, & \nu=0, \\
0, & g \in V_{m}(p) \backslash \theta, & \nu=0, \\
1, & g \in V_{m}(p), & \nu=1, \ldots, p^{m}-2,
\end{array}\right. \\
& \chi_{U}\left(\widetilde{g} \cdot \alpha^{\nu}\right)=\left\{\begin{array}{rll}
p^{m}-1, & g=\theta, & \nu=0, \\
-1, & g \in V_{m}(p) \backslash \theta, & \nu=0, \\
0, & g \in V_{m}(p), & \nu=1, \ldots, p^{m}-2 .
\end{array}\right.
\end{aligned}
$$

Вычислив скалярные произведения, получим

$$
\left(\chi_{Q}, \chi_{U}\right)=\left(\chi_{U}, \chi_{U}\right)=1
$$

Следовательно, $U$ - неединичная неприводимая компонента представления $Q$. Будем считать, что $U_{1}=U$, при этом $k^{(1)}=1$. Из соотношения (19) с учетом свойства мультипликативности евклидовой нормы матриц следует неравенство

$$
\delta_{N}^{2} \leqslant \epsilon\left(\Phi_{\eta_{1}}^{(q)}\left(U_{1}\right)\right)^{2 N}
$$

Определим числа $\nu_{i} \in\left\{0, \ldots, p^{m}-2\right\}$ равенствами $\alpha_{i}=\alpha^{\nu_{i}}, i=1, \ldots, s$. Согласно (17)

$$
\Phi_{\eta_{1}}^{(q)}\left(U_{1}\right)=\left[\Phi_{\eta_{1}}^{(q)}\left(U_{1}\right)_{l, j}\right]_{l, j=1}^{p^{m_{-}-1}}=\sum_{i=1}^{s} \sum_{g \in V_{m}(p)} p_{g, i} \cdot U\left(\widetilde{g} \cdot \alpha^{\nu_{i}}\right) .
$$

Согласно (28), для любых $l, j=1, \ldots, p^{m}-1$ элемент матрицы

$$
\Phi_{\eta_{1}}^{(q)}\left(U_{1}\right)_{l, j}=\sum_{i=1}^{s} \sum_{g \in V_{m}(p)} p_{g, i} \cdot U\left(\widetilde{g} \cdot \alpha^{\nu_{i}}\right)_{l, j}
$$

ненулевой, если и только если существует (и притом единственное) число $i \in\{1, \ldots, s\}$, для которого $j \equiv l+\nu_{i}\left(\bmod \left(p^{m}-1\right)\right)$. 
В этом случае

$$
\Phi_{\eta_{1}}^{(q)}\left(U_{1}\right)_{l, j}=\sum_{g \in V_{m}(p)} p_{g, i} \cdot \psi_{l}(g)
$$

Следовательно,

$$
\begin{aligned}
\epsilon\left(\Phi_{\eta_{1}}^{(q)}\left(U_{1}\right)\right)^{2} & =\sum_{l=1}^{p^{m}-1} \sum_{i=1}^{s}\left(\sum_{g \in V_{m}(p)} p_{g, i} \cdot \psi^{(l)}(g)\right)\left(\sum_{h \in V_{m}(p)} p_{h, i} \cdot \overline{\psi^{(l)}(h)}\right)= \\
& =\sum_{i=1}^{s} \sum_{g \in V_{m}(p)} \sum_{h \in V_{m}(p)} \sum_{l=1}^{p^{m}-1} p_{g, i} \cdot p_{h, i} \cdot \psi^{(l)}(g-h) .
\end{aligned}
$$

Из равенства (27) и соотношений ортогональности для характеров группы $V_{m}(p)$ следует, что

$$
\sum_{l=1}^{p^{m}-1} \psi^{(l)}(g-h)=\left\{\begin{array}{rr}
p^{m}-1, & g=h, \\
-1, & g \neq h .
\end{array}\right.
$$

Используя эти равенства, получим

$$
\begin{aligned}
\epsilon\left(\Phi_{\eta_{1}}^{(q)}\left(U_{1}\right)\right)^{2} & =\sum_{i=1}^{s} \sum_{g \in V_{m}(p)}\left(p_{g, i}^{2} \cdot\left(p^{m}-1\right)-\sum_{h \in V_{m}(p) \backslash g} p_{g, i} \cdot p_{h, i}\right)= \\
& =p^{m} \sum_{i=1}^{s} \sum_{g \in V_{m}(p)} p_{g, i}^{2}-\sum_{i=1}^{s} \sum_{g, h \in V_{m}(p)} p_{g, i} \cdot p_{h, i}= \\
& =p^{m} \sum_{i=1}^{s} \sum_{g \in V_{m}(p)} p_{g, i}^{2}-\sum_{i=1}^{s} q_{i}^{2} .
\end{aligned}
$$

Итак,

$$
\epsilon\left(\Phi_{\eta_{1}}^{(q)}\left(U_{1}\right)\right)^{2}=\rho
$$

Соотношение (10) следует из (29) и (30).

Автор благодарен А.В. Лапшину за внимательное прочтение работы и полезные замечания.

2017, T. 8, № 1, C. $31-50$ 


\section{Список литературы}

[1] Глухов М. М., “О числовых параметрах, связанных с заданием конечных групп системами образующих элементов”, Труды по дискретной математике, 1, М.: Научное изд-во ТВП, 1997, 43-66.

[2] Горчинский Ю.Н., Круглов И.А., Капитонов В.М., “Вопросы теории распределений на конечных группах”, Труды по дискретной математике, 1, М.: Научное изд-во ТВП, 1997, $85-112$.

[3] Круглов И.А., “Условия предельной равновероятности распределений в схеме линейной авторегрессии со случайным управлением на конечной группе”, Дискретн. матем., 17:3 (2005), 12-18.

[4] Круглов И. А., “О слоях в системе образующих элементов подпрямого произведения подгрупп конечной группы”, Дискретн. матем., 21 :1 (2009), 52-65.

[5] Круглов И. А., “Случайные последовательности вида $X_{t+1}=a_{t} \cdot X_{t}+b_{t}(\bmod n)$ с зависимыми коэффициентами $a_{t}, b_{t}$ ”, Дискретн. матем., 17:2 (2005), 49-55.

[6] Diaconis P., Group Representations in Probability and Statistics, Lecture Notes-Monograph Series, 11, Hayward, CA: Inst. of Math. Statist., 1988, 198 pp.

[7] Hildebrand M., "Random processes of the form $X_{n=1}=a_{n} X_{n}+b_{n}(\bmod p)$ ", Ann. Probab., $21: 2$ (1993), 710-720.

[8] Hildebrand M., "Random processes of the form $X_{n=1}=a_{n} \cdot X_{n}+b_{n}(\bmod p)$, where $b_{n}$ takes on a single value". In: "Random Discrete Structures", IMA Vol. Math. Appl., 76, Heidelberg etc. : Springer, 1996, 153-174.

[9] Asci C., "Generating uniform random vectors", J. Theoret. Probab., $14: 2$ (2001), 333-356.

[10] Helleloid G., Automorphism Groups of Finite p-groups: Structure and Applications, Ph.D., The Univ. of Texas at Austin, 2007, 107 pp., http://arxiv.org/math.GR/07112816 math.GR/07112816

[11] Bianko S., Random processes of the form $X_{n=1}=A_{n} \cdot X_{n}+B_{n}(\bmod p)$ in two dimensions, Ph.D., State Univ. of New York at Albany, 2012, 37 pp.

[12] Hildebrand M., "A lower bound for the Chung-Diaconis-Graham random process", Proc. Amer. Math. Soc., 137:4 (2009), 1479-1487.

[13] Hildebrand M., McCollum J., "Generating random vectors in $(Z / p Z)^{d}$ via an affine random process", J. Theor. Probab., $21: 4$ (2008), 802-811. 